\title{
Investigating the causal nature of the relationship of subcortical brain volume with smoking and alcohol use
}

Emma Logtenberg*, Martin F. Overbeek*, Joëlle A. Pasman, Abdel Abdellaoui, Maartje Luijten, Ruth J. van Holst, Jacqueline M. Vink, Damiaan Denys, Sarah E. Medland, Karin J. H. Verweij and Jorien L. Treur

\section{Background}

Structural variation in subcortical brain regions has been linked to substance use, including the most commonly used substances nicotine and alcohol. Pre-existing differences in subcortical brain volume may affect smoking and alcohol use, but there is also evidence that smoking and alcohol use can lead to structural changes.

\section{Aims}

We assess the causal nature of the complex relationship of subcortical brain volume with smoking and alcohol use, using bidirectional Mendelian randomisation.

\section{Method}

Mendelian randomisation uses genetic variants predictive of a certain 'exposure' as instrumental variables to test causal effects on an 'outcome'. Because of random assortment at meiosis, genetic variants should not be associated with confounders, allowing less biased causal inference. We used summary-level data of genome-wide association studies of subcortical brain volumes (nucleus accumbens, amygdala, caudate, hippocampus, pallidum, putamen and thalamus; $n=50290$ ) and smoking and alcohol use (smoking initiation, $n=848460$; cigarettes per day, $n=216$ 590; smoking cessation, $n=378249$; alcoholic drinks per week, $n=630$ 154; alcohol dependence, $n=46$ 568). The main analysis, inverse-variance weighted regression, was verified by a wide range of sensitivity methods.

\section{Results}

There was strong evidence that liability to alcohol dependence decreased amygdala and hippocampal volume, and smoking more cigarettes per day decreased hippocampal volume. From subcortical brain volumes to substance use, there was no or weak evidence for causal effects.

\section{Conclusions}

Our findings suggest that heavy alcohol use and smoking can causally reduce subcortical brain volume. This adds to accumulating evidence that alcohol and smoking affect the brain, and likely mental health, warranting more recognition in public health efforts.

\section{Keywords}

Smoking; alcohol use; alcohol dependence; subcortical brain volume; Mendelian randomisation.

\section{Copyright and usage}

(C) The Author(s), 2021. Published by Cambridge University Press on behalf of the Royal College of Psychiatrists. This is an Open Access article, distributed under the terms of the creative Commons Attribution-NonCommercial-NoDerivatives licence (http://creativecommons.org/licenses/by-nc-nd/4.0/), which permits non-commercial re-use, distribution, and reproduction in any medium, provided the original work is unaltered and is properly cited. The written permission of Cambridge University Press must be obtained for commercial re-use or in order to create a derivative work.
Subcortical brain regions have consistently been implicated in substance use, playing a crucial role in the brain's reward system. Addiction is thought to reflect a vicious cycle of intoxication, withdrawal and craving, with (subcortical) brain circuits mediating these stages. ${ }^{1}$ The causal nature of the relationship between structural variation in subcortical brain regions and substance use is unclear. Subcortical brain volume and substance use are heritable, and there is evidence that they share (part of) their genetic aetiology. ${ }^{2}$ Alternatively, the relationship may be causal, with pre-existing differences in subcortical brain volume asserting a direct effect on substance use. Causality in the other direction is also plausible, i.e. substance use affecting brain structure. Most likely, the relationship between subcortical brain volume and substance use reflects a combination of mechanisms.

The majority of substance use-related morbidity and mortality result from smoking and alcohol use. ${ }^{3}$ Literature on the relationship of subcortical brain volumes with smoking and alcohol use comprises predominantly small cross-sectional studies, reporting mixed findings. Smoking has been associated with smaller nucleus accumbens, ${ }^{4}$ amygdala, ${ }^{5,6}$ hippocampus, ${ }^{7}$ pallidum ${ }^{6}$ and thalamus ${ }^{6}$ volumes, with smaller ${ }^{5}$ and larger ${ }^{8}$ caudate volume, and larger

* Joint first authorship. putamen volume. ${ }^{4}$ Alcohol use has been associated with smaller nucleus accumbens, ${ }^{9}$ amygdala, ${ }^{10}$ hippocampus ${ }^{9}$ and pallidum volumes, ${ }^{9}$ and with smaller ${ }^{11}$ and larger ${ }^{12}$ caudate, smaller ${ }^{9}$ and larger $^{13}$ thalamus, and smaller ${ }^{9}$ and larger ${ }^{13}$ putamen volumes. Recently, the Enhancing Neuro Imaging Genetics Through Meta Analysis (ENIGMA) addiction working group attempted to resolve these inconsistent findings with a mega-analysis of subcortical thickness and surface area, among 1628 controls and 2277 individuals dependent on alcohol, nicotine, cocaine, methamphetamine and/or cannabis. ${ }^{14}$ Smoking was associated with greater thickness and surface area, most notably for the nucleus accumbens and hippocampus, whereas alcohol dependence was associated with lower thickness and surface area, most notably for the hippocampus, amygdala, thalamus and putamen.

\section{Identifying causal relationships}

Longitudinal studies investigating subcortical brain volume and substance use are scarce. One recent study in 714 individuals at ages 14 and 19 years showed that alcohol and cannabis use were associated with accelerated cortical thinning and a (mild) increase in subcortical volumes. ${ }^{15}$ Although these were longitudinal analyses, the observational design leaves potential for bias from 
(unmeasured) confounding and reverse causality. A randomised controlled trial would be unfeasible for the relationship under study, but Mendelian randomisation provides an alternative. ${ }^{16}$ Instead of experimental manipulation, Mendelian randomisation uses genetic variants as proxies for the independent variable. Because genes are randomly transmitted from parents to offspring, genetic variants should not be associated with confounders. Reverse causation is not likely either, as the genetic independent variable is fixed at birth. A recent Mendelian randomisation study found no evidence for a causal effect of smoking on hippocampal volume, ${ }^{17}$ but the analyses were based on much smaller samples than currently available and other subcortical regions were not included. We conduct the first comprehensive Mendelian randomisation study, using the largest genetic data-sets available of the volume of seven subcortical regions (nucleus accumbens, amygdala, caudate nucleus, hippocampus, pallidum, putamen and thalamus) and substance use (smoking initiation, cigarettes per day, smoking cessation, alcoholic drinks per week and alcohol dependence), to probe bi-directional, causal relationships.

\section{Method}

\section{Mendelian randomisation}

Mendelian randomisation is based on the premise that genetic markers can be used as proxies for a variable hypothesised to be a risk factor, or 'exposure', for another 'outcome' variable. Single nucleotide polymorphisms (SNPs) are most often used. The validity of Mendelian randomisation relies on three assumptions: the association of the genetic instrument with the exposure is robust (ensured by selecting SNPs under $P<5 \mathrm{e}-08$ ); the instrument is not associated with confounding variables and the instrument does not influence the outcome through any other path than the exposure. Horizontal pleiotropy, where an SNP directly affects multiple traits, could lead to the second and third assumptions being violated. To assess whether the assumptions were met, we applied a wide range of sensitivity methods.

\section{Data}

All data-sets and measures used for our analyses are visually depicted in Supplementary Fig. 1 available at https://doi.org/10. 1192/bjp.2021.81, and described in more detail in Supplementary Table 1. For subcortical brain volumes, we used summary-level data from a published genome-wide association study (GWAS) $\left(n=13171^{18}\right)$, and meta-analysed these with summary-level data from a GWAS of 37119 UK Biobank participants (Supplementary Appendix 1). This resulted in a sample of 50290 for nucleus accumbens, amygdala, caudate nucleus, hippocampus, pallidum, putamen and thalamus volumes. For substance use, we obtained summarylevel data from the single largest GWAS on smoking and alcohol use. ${ }^{19}$ To prevent sample overlap, we excluded UK Biobank data from this GWAS. We meta-analysed the remaining summary statistics with those from 23andMe (separately obtained from 23andMe, as these are not publicly available), resulting in sample sizes of $n=$ 848460 for smoking initiation, $n=216590$ for cigarettes per day, $n=378249$ for smoking cessation and $n=630154$ for alcoholic drinks per week. We also obtained summary-level data from a separate GWAS on alcohol dependence $(n=46568) .{ }^{20}$ Both the subcortical brain volumes and substance use meta-analyses were $\mathrm{N}$-weighted, owing to measurement variance in the original samples, resulting in $z$-scores. To allow Mendelian randomisation analysis, we constructed beta-coefficients and standard errors using these $z$-scores, effect allele frequencies and sample size. ${ }^{21}$ Although the unit of Mendelian randomisation estimates based on constructed beta-coefficients and s.e. cannot be reliably interpreted, the direction of effect and statistical strength is robust.

Because we obtained the exposure and outcome estimates from separate samples, it is not possible to verify if individuals in the outcome sample were affected by the exposure. Therefore, when we refer to an exposure causally affecting an outcome, this should be interpreted as an effect of the liability to that exposure. We assessed smoking and alcohol use in 37119 UK Biobank participants included in the subcortical brain volume GWAS, to confirm that smoking and alcohol use were prevalent (Supplementary Table 2).

All procedures contributing to this work comply with the ethical standards of the relevant national and institutional committees on human experimentation and with the Helsinki Declaration of 1975, as revised in 2008. Summary-level data from consortia were used, with individual sites having obtained ethical approval and informed consent from participants. For UK Biobank (http:// biobank.ctsu.ox.ac.uk/crystal/field.cgi?id=200), the National Health Service North West Centre for Research Ethics Committee provided ethical approval (reference umber 11/NW/0382).

\section{Main analysis}

We explain the analysis by using an example where hippocampal volume is the exposure (the independent variable) and smoking initiation is the outcome (the dependent variable). First, SNPs that robustly predict hippocampal volume $(P<5 \mathrm{E}-08)$ were identified in the hippocampus GWAS, and their effect size estimates and standard errors were extracted. These SNPs - the genetic instrument - were then identified in the smoking initiation GWAS, and their effect estimates and standard errors were extracted. To estimate the causal effect, the SNP-smoking initiation association was divided by the SNP-hippocampal volume association for each SNP separately. To reach a combination of effects with minimum variance for all SNPs combined, the individual SNP effects were weighted by the inverse of their variance. Inverse-variance weighted regression (IVW) provides the first indication of causality, assuming that all Mendelian randomisation assumptions are met.

We tested causal relationships with subcortical brain volumes as exposures and smoking initiation, cigarettes per day, smoking cessation, alcoholic drinks per week and alcohol dependence as outcomes, and vice versa, with smoking initiation, alcoholic drinks per week and alcohol dependence as exposures and subcortical volumes as outcomes. If fewer than ten SNPs with $P<5 e-08$ were available, we additionally constructed an instrument containing SNPs under a more lenient threshold of $P<1 \mathrm{e}-05$. We clumped SNPs for independence at $r^{2}<0.01$ and $10000 \mathrm{~kb}^{22}$

Because the GWAS for cigarettes per day consisted of smokers only, ${ }^{19}$ SNPs from that study cannot be used as proxies for cigarettes per day in never smokers. Therefore, the complete subcortical brain volume data-set $(n=50290)$, consisting of smokers and never smokers, could not be used. For UK Biobank participants $(n=37119)$, we could obtain information on smoking and perform GWAS of subcortical brain volumes in never smokers $(n=22555)$ and ever smokers $(n=14564)$. We then applied summary-level Mendelian randomisation with cigarettes per day as the exposure, in never and ever smokers separately. If the genetic instrument for cigarettes per day predicts subcortical brain volume in never smokers, this indicates horizontal pleiotropy because there cannot be a true causal effect. ${ }^{23}$ Note that a similar stratification approach was not indicated for alcohol use, as the exposure sample from which the genetic instruments for alcoholic drinks per week were obtained included non-drinkers. Unlike for smoking, almost all participants in the European samples used are expected to have been exposed to (some) alcohol. Also note 
that all GWAS included in our analyses corrected for age and gender (Supplementary Table 2).

\section{Sensitivity analyses}

The F-statistic was computed to assess instrument strength for all exposures, with $F>10$ reflecting a sufficiently strong instrument. ${ }^{24}$ To test the robustness of IVW, we applied six sensitivity methods with different, partly contrasting assumptions. First, we used weighted median regression, which produces a reliable causal estimate if $\geq 50 \%$ of the total weight of the genetic instrument comes from valid (unbiased) SNPs. ${ }^{25}$ Second, we used weighted mode regression, which clusters SNPs in the genetic instrument and selects the SNP cluster with the largest weight as the final causal estimate. This results in an unbiased value if the most common causal estimate is indeed the true causal effect. ${ }^{26}$ Third, we used Mendelian randomisation-Egger (MR-Egger), which permits the intercept to deviate from zero, allowing a formal test of horizontal pleiotropy (when there is no horizontal pleiotropy, the intercept is zero). ${ }^{27}$ MR-Egger is reliable if the instrument strength independent of direct effect (InSIDE) assumption is met, meaning that the strength of the instrument (SNP-exposure association) should not correlate with the direct effect of the SNPs on the outcome. MR-Egger also requires sufficiently strong instruments, indicated as the no measurement error (NOME) assumption. This can be assessed with the IGX2 statistic, ranging between 0 and 1 . A lower value reflects a higher chance that NOME is violated. ${ }^{28}$ If IGX2 $\geq 0.9$, NOME is unlikely to be violated and results are reliable. If IGX2 is within 0.6-0.9, NOME may have been violated but MR-Egger simulation extrapolation (SIMEX) can correct for this. If IGX2 $<0.6$, MREgger results are likely biased. Fourth, we used generalised summary-data-based Mendelian randomisation (GSMR), which accounts for very low levels of linkage disequilibrium between SNPs and sampling variance in the estimated SNP effects, to attain higher statistical power. GSMR filters outlier SNPs based on their effect size (heterogeneity in dependent instruments (HEIDI) filtering). ${ }^{29}$ Fifth, we used Mendelian randomisation pleiotropy residual sum and outlier (MR-PRESSO), which compares the observed residual sum of squares with the expected residual sum of squares for each SNP, re-running outlier-corrected IVW analyses. ${ }^{30}$ Sixth, we used Steiger filtering, which identifies potential bias from reverse causation. It calculates how much variance each SNP explains in the exposure and the outcome, and tests whether the explained variance is, as expected, higher for exposure than outcome. SNPs that explain more variance in the outcome than the exposure are excluded. ${ }^{31}$

We computed Cochran's Q-statistic to assess heterogeneity across the causal estimates of SNPs in each instrument ${ }^{24}$; high heterogeneity points to horizontal pleiotropy. It is also possible for a true causal effect to run through multiple, separate biological pathways, resulting in heterogeneity. To assess variability in the power of the genetic instruments, we computed the amount of variance that each instrument explained in the proposed exposure variable. $^{32}$

Analyses were conducted in $\mathrm{R}$ version 4.0 .3 for Windows (see see https://cran.r-project.org/bin/windows/base/old/4.0.3/), using TwoSampleMR, ${ }^{22}$ GSMR $^{29}$ and MR-PRESSO ${ }^{30}$ packages.

\section{Appraisal of the evidence}

We did not correct for multiple testing explicitly because we analyse phenotypes for which, a priori, there are plausible hypotheses why they are (causally) associated and we want to avoid appraising evidence based on an arbitrary threshold. Rather than using a strict significance threshold, we ascribe a finding as showing strong evidence, evidence, weak evidence or no clear evidence for a causal effect, based on both the IVW result (adhering to the interpretation of $P$-values suggested by Sterne et $\mathrm{al}^{33}{ }^{33}$ who stressed the importance of considering $P$-values and evidence on a spectrum, rather than in discrete terms) and the sensitivity analyses. This approach provides a safeguard against chance findings, since a finding is only considered robust when the main result is corroborated by sensitivity analyses. A similar approach has previously been adopted by other, high-impact Mendelian randomisation studies. ${ }^{34,35}$ Note that because sensitivity methods rely on stricter assumptions than IVW, their statistical power is lower. It is to be expected that the statistical evidence, but not the effect size, decreases with stricter sensitivity methods, even for a true causal effect.

\section{Results}

All genetic instruments showed sufficient strength (Supplementary Tables 3-5). The amount of variance that the genetic instruments explained in the corresponding subcortical region and substance use variables, as well as the estimated SNP heritability for each variable are presented in Table 1. Figure 1 displays all relationships with (weak-to-strong) evidence for causality.

Table 1 Explained variance for the genetic instruments used for Mendelian randomisation analyses and SNP-based heritability estimates for all phenotypes, based on full summary statistics

\begin{tabular}{|c|c|c|c|c|}
\hline Variable & Threshold & $\begin{array}{l}\text { Number of SNPs in genetic } \\
\text { instrument }\end{array}$ & $\begin{array}{l}\text { Percentage of variance that the genetic instrument explains } \\
\text { in the variable }\end{array}$ & $\begin{array}{l}\text { SNP-based } \\
\text { heritability }\end{array}$ \\
\hline Nucleus accumbens & $5.00 \mathrm{E}-08$ & 13 & $0.70 \%$ & $16.70 \%^{a}$ \\
\hline Amygdala & $5.00 \mathrm{E}-08$ & 2 & $0.17 \%$ & $9.26 \%^{a}$ \\
\hline Amygdala & $1.00 \mathrm{E}-05$ & 40 & $0.97 \%$ & $9.26 \%$ \\
\hline Caudate & $5.00 \mathrm{E}-08$ & 53 & $3.97 \%$ & $31.66 \%^{a}$ \\
\hline Hippocampus & $5.00 \mathrm{E}-08$ & 19 & $1.60 \%$ & $17.24 \%^{a}$ \\
\hline Pallidum & 5.00E-08 & 25 & $1.82 \%$ & $18.44 \%^{a}$ \\
\hline Putamen & 5.00E-08 & 35 & $2.18 \%$ & $27.65 \%^{a}$ \\
\hline Thalamus & $5.00 E-08$ & 12 & $0.70 \%$ & $20.63 \%^{a}$ \\
\hline Smoking initiation & $5.00 \mathrm{E}-08$ & 346 & $1.43 \%$ & $7.77 \%^{\mathrm{b}}$ \\
\hline Cigarettes per day & $5.00 \mathrm{E}-08$ & 49 & $1.27 \%$ & $8.04 \%^{\mathrm{b}}$ \\
\hline $\begin{array}{l}\text { Alcoholic drinks per } \\
\quad \text { week }\end{array}$ & $5.00 \mathrm{E}-08$ & 92 & $0.56 \%$ & $4.19 \%^{\mathrm{b}}$ \\
\hline Alcohol dependence & $5.00 \mathrm{E}-08$ & 10 & $0.59 \%$ & $9.00 \%^{b}$ \\
\hline
\end{tabular}




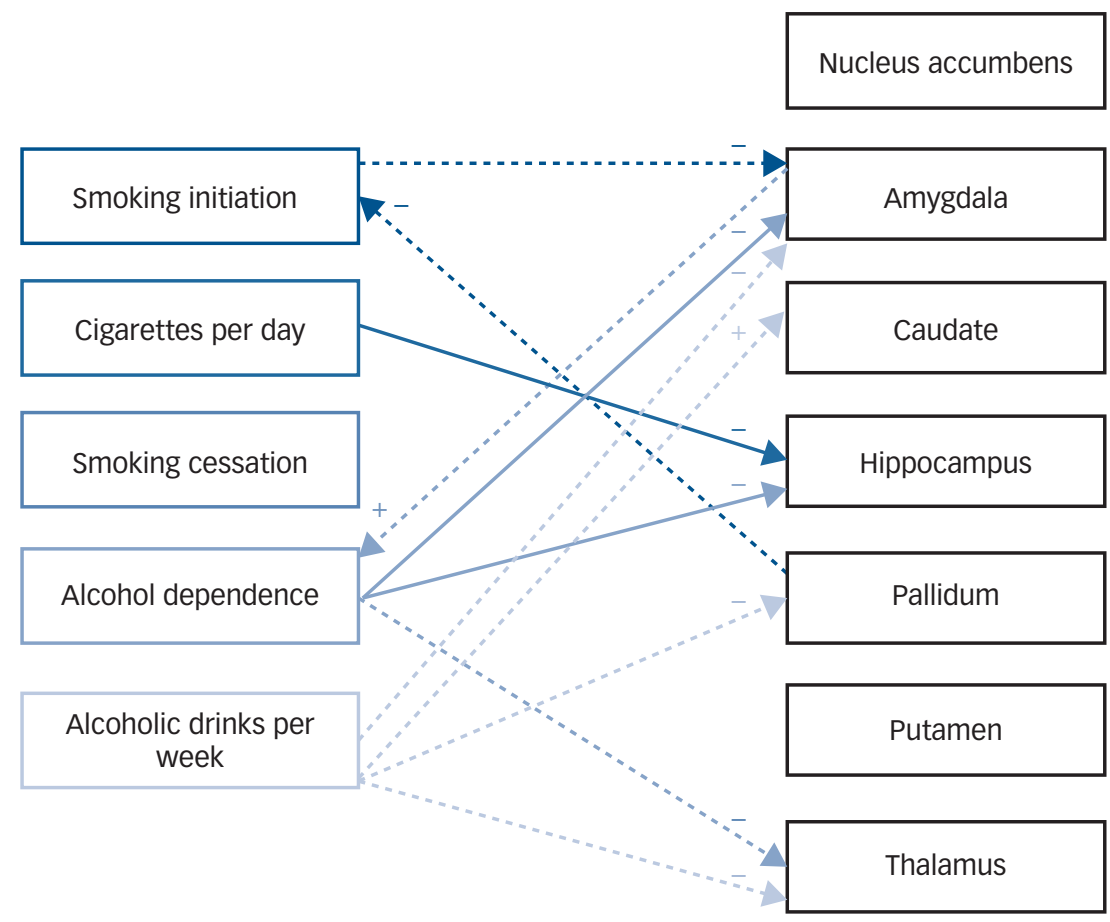

Fig. 1 Graphical display of the relationships with evidence for causality. Minus (-) signifies a negative, decreasing effect; plus (+) signifies a positive, increasing effect. The thicker lines reflect evidence or strong evidence for causality; the dotted, thinner lines signify weak evidence for causality. Note that causal effects were tested in both directions for all relationships except for smoking cessation, which was only tested as an outcome variable.

\section{Causal relationships from subcortical volumes to substance use}

There was weak evidence that a larger pallidum volume decreases the odds of initiating smoking $\left(\beta_{\mathrm{IVW}}=-0.04, \quad P=0.053\right)$. Weighted median, weighted mode and GSMR corroborated this, showing similar effect sizes and stronger statistical evidence (Table 2). Although there was no clear evidence for horizontal pleiotropy (MR-Egger intercept $-0.003, P=0.332$; Supplementary Table 6), the regression coefficient of MR-Egger did not indicate a causal effect (Table 2). There was strong evidence for heterogeneity among the SNP effects (Cochran's $Q$-statistic $P=2.4 \mathrm{E}-05$; Supplementary Table 7). MR-PRESSO identified two SNP outliers but there was no distortion of the causal estimate after outlier removal (Supplementary Table 8). Steiger filtering did not identify SNPs that explained more variance in the outcome than the exposure (Supplementary Table 9).

There was weak evidence that a larger amygdala volume increases alcohol dependence risk $\left(P<1 \mathrm{E}-05, \beta_{\mathrm{IVW}}=0.08, P=\right.$ 0.046 ), corroborated by weighted median, weighted mode and GSMR, but not MR-Egger. There was no clear evidence for horizontal pleiotropy (MR-Egger intercept $0.003, P=0.400$ ) or heterogeneity $(P=0.621)$. MR-PRESSO did not identify SNP outliers, nor did Steiger filtering identify SNPs that explained more variance in the outcome than the exposure. With a two-SNP instrument $(P<$ $5 \mathrm{E}-08)$, there was a similar sized positive effect, but no clear statistical evidence $\left(\beta_{\mathrm{IVW}}=0.09, P=0.522\right)$.

There was very weak evidence that a larger amygdala volume increased the number of alcoholic drinks per week $\left(\beta_{\mathrm{IVW}}=0.06\right.$, $P=0.098$ ), but sensitivity analyses were not possible because the $P<5 \mathrm{E}-08$ instrument only contained two SNPs. With 40 SNPs under $P<1 \mathrm{E}-05$, there was no clear evidence for an effect $\left(\beta_{\mathrm{IVW}}\right.$ $=0.01, P=0.289$ ).

No other analyses showed clear evidence for causal effects.

\section{Causal relationships from substance use to subcortical volumes}

There was weak evidence that smoking initiation decreases amygdala volume $\left(\beta_{\mathrm{IVW}}=-0.05, P=0.046\right)$, confirmed by GSMR (Table 3 ). There was no clear evidence for horizontal pleiotropy (MR-Egger intercept $-0.001, P=0.457$ ), but strong evidence for heterogeneity (Cochran's $Q$-statistic $P=2.4 \mathrm{E}-07$ ). MR-PRESSO identified one SNP outlier, which did not affect the results. Steiger filtering excluded 44 SNPs, but after running the analyses with the 302 remaining SNPs, evidence for a causal effect remained $\left(\beta_{\mathrm{IVW}}=-0.06, P=0.013\right)$.

In the analyses stratified for smoking status, there was strong evidence that smoking more cigarettes per day decreases hippocampal volume in smokers $\left(\beta_{\mathrm{IVW}}=-94.73, P=1.8 \mathrm{E}-06\right)$. Results were consistent with weighted median, weighted mode, MR-Egger and GSMR, albeit with a smaller effect size for the latter. There was no clear evidence for horizontal pleiotropy (MR-Egger intercept $0.633, P=0.568)$ or heterogeneity $(P=0.357)$. No SNP outliers were identified with MR-PRESSO. Steiger filtering identified nine SNPs, but after excluding these (leaving 40 SNPs), strong and consistent evidence for causality remained. There was weak evidence for a negative effect of cigarettes per day on hippocampal volume in never smokers (indicating horizontal pleiotropy), with a much smaller, less significant effect size $\left(\beta_{\mathrm{IVW}}=-30.40, P=0.050\right)$ and no consistency across sensitivity methods. Since the effect size and statistical evidence were substantially larger and stronger in ever compared with never smokers (Supplementary Fig. 2), there is likely a negative causal effect of smoking heaviness on hippocampal volume (horizontal pleiotropy notwithstanding).

There was evidence for a negative effect of cigarettes per day on putamen volume, but this relationship seems pleiotropic. The effect size and statistical evidence in ever and never smokers is nearly identical $\left(\beta_{\mathrm{IVW}}=-68.86, P=0.018\right.$ and $\beta_{\mathrm{IVW}}=-71.41, P=0.003$, 
Table 2 Mendelian randomisation analysis, with subcortical brain volumes as the exposures and smoking and alcohol use as the outcomes

\begin{tabular}{|c|c|c|c|c|c|c|c|c|c|c|c|c|c|c|c|c|c|c|}
\hline \multirow[b]{2}{*}{ Exposure } & \multirow[b]{2}{*}{ Outcome } & \multirow{2}{*}{$\begin{array}{c}\text { SNP } \\
n\end{array}$} & \multicolumn{3}{|c|}{ IVW } & \multicolumn{3}{|c|}{ Weighted median } & \multicolumn{3}{|c|}{ Weighted mode } & \multicolumn{3}{|c|}{ MR-Egger ${ }^{\mathrm{a}}$} & \multirow{2}{*}{$\begin{array}{c}\text { SNP } \\
n\end{array}$} & \multicolumn{3}{|c|}{ GSMR } \\
\hline & & & $\beta$-value & $95 \% \mathrm{Cl}$ & $P$-value & $\beta$-value & $95 \% \mathrm{Cl}$ & $P$-value & $\beta$-value & $95 \% \mathrm{Cl}$ & $P$-value & $\beta$-value & $95 \% \mathrm{Cl}$ & $P$-value & & $\beta$-value & $95 \% \mathrm{Cl}$ & $P$-value \\
\hline Nucleus accumbens & Smoking initiation & 13 & 0.02 & -0.05 to 0.09 & 0.542 & -0.01 & -0.08 to 0.06 & 0.789 & $-4.3 \mathrm{E}-04$ & -0.12 to 0.12 & 0.994 & -0.24 & -0.37 to -0.11 & 0.003 & 13 & 0.02 & -0.03 to 0.06 & 0.519 \\
\hline Amygdala $P<5 \mathrm{E}-08$ & Smoking initiation & 2 & 0.10 & -0.12 to 0.31 & 0.388 & - & - & - & - & - & - & - & - & - & - & - & - & - \\
\hline Amygdala $P<1 \mathrm{E}-05$ & Smoking initiation & 40 & -0.01 & -0.04 to 0.03 & .770 & $3.6 \mathrm{E}-03$ & -0.04 to 0.04 & 0.864 & $1.0 \mathrm{E}-03$ & -0.04 to 0.04 & 0.957 & 0.04 & -0.01 to 0.10 & 0.134 & 40 & $5.6 \mathrm{E}-04$ & -0.03 to 0.03 & 0.966 \\
\hline Caudate & Smoking initiation & 53 & -0.02 & -0.05 to 0.02 & 0.298 & $-5.8 \mathrm{E}-03$ & -0.04 to 0.03 & 0.740 & $-2.1 \mathrm{E}-03$ & -0.05 to 0.04 & 0.931 & 0.05 & -0.06 to 0.17 & 0.352 & 49 & 4.3E-03 - & -0.02 to 0.03 & 0.702 \\
\hline Hippocampus & Smoking initiation & 19 & 0.02 & -0.07 to 0.01 & 0.644 & 0.02 & -0.05 to 0.09 & 0.545 & 0.04 & -0.10 to 0.17 & 0.599 & -0.30 & -0.81 to 0.21 & 0.271 & 19 & $5.4 \mathrm{E}-04$ & -0.04 to 0.04 & 0.978 \\
\hline Pallidum & Smoking initiation & 25 & -0.04 & -0.08 to $0.07 \mathrm{E}-04$ & 0.053 & -0.05 & -0.09 to -0.01 & 0.024 & -0.07 & -0.12 to -0.03 & 0.006 & $1.8 \mathrm{E}-03$ & -0.09 to 0.09 & 0.970 & 23 & -0.05 & -0.07 to -0.02 & 0.001 \\
\hline Putamen & Smoking initiation & 35 & $-3.8 \mathrm{E}-03$ & -0.05 to 0.04 & 0.859 & -0.02 & -0.05 to 0.01 & 0.200 & -0.02 & -0.05 to 0.01 & 0.266 & 0.02 & -0.08 to 0.11 & 0.745 & 29 & $1.8 \mathrm{E}-03$ & -0.02 to 0.02 & 0.880 \\
\hline Thalamus & Smoking initiation & 12 & -0.05 & -0.16 to 0.05 & 0.291 & -0.06 & -0.14 to 0.03 & 0.177 & -0.06 & -0.20 to 0.08 & 0.395 & -0.16 & -0.36 to 0.04 & 139 & 10 & 3.5E-03 & -0.06 to 0.05 & 0.903 \\
\hline Nucleus accumbens & Smoking ces & 13 & 0.02 & -0.02 to 0 & & 0.02 & -0.03 to 0.07 & 0.477 & 0.04 & -0.03 to 0.11 & 0.304 & 0.06 & -0.04 to 0.17 & 0.265 & 13 & -0.02 & -0.06 to 0.02 & 0.303 \\
\hline Amygda & Smoking cessation & 2 & -0.14 & -0.32 to 0.04 & 31 & - & - & - & - & - & - & - & - & - & - & - & - & - \\
\hline Amygdala & Smoking ces & 39 & -0.01 & -0.04 to $c$ & & -0.03 & -0.07 to 0.01 & 0.150 & -0.02 & -0.08 to 0.04 & 0.501 & -0.07 & -0.19 to 0.05 & 2.309 & 39 & 0.01 & -0.01 to 0.04 & 0.348 \\
\hline Caudate & Smoking cessation & 53 & 0.01 & -0.02 to 0.04 & 706 & $-2.4 \mathrm{E}-03$ & -0.04 to 0.03 & 0.886 & -0.01 & -0.07 to 0.05 & 0.848 & $-8.5 E-03$ & -0.09 to 0.07 & 831 & 50 & -0.01 & -0.03 to 0.01 & 0.389 \\
\hline Hippocampus & Smoking & 19 & 0.0 & 04 to & & -0.01 & -0.06 to 0.04 & & -0.03 & -0.12 to & 23 & -0.03 & -0.27 to 0.2 & 800 & 19 & -0. & -0.05 to & 0.528 \\
\hline Pallidum & Smoking C & 25 & 0.02 & -0.01 to 0 & 10 & 0.01 & -0.03 to 0.05 & 660 & 0.01 & -0.04 to 0.05 & 0.781 & -0.0 & -0.08 to 0.05 & 635 & 25 & -0. & -0.05 to 0.00 & 0.061 \\
\hline Putamen & Smoking cessation & 35 & 0.01 & -0.02 to 0.05 & & $8.5 \mathrm{E}-03$ & -0.03 to 0.05 & 0.660 & 0.0 & -0.05 to 0.06 & 0.797 & -0.03 & -0.12 to 0.06 & 532 & 35 & -0.01 & -0.03 to 0.01 & 0.355 \\
\hline Thalamus & Smoking cessation & 13 & 3.7E-03 & -0.06 to 0.07 & 0.907 & 4.3E-03 & -0.06 to 0.06 & 0.889 & 0.01 & -0.07 to 0.08 & 0.864 & -0.10 & -0.31 to 0.11 & 361 & 13 & $2.9 E-03$ & -0.04 to 0.04 & 0.893 \\
\hline Nucleus accumbens & CPD & 13 & 0.04 & -0.01 to 0.09 & & 0.04 & -0.02 to 0.11 & 0.198 & 0.06 & -0.05 to 0.16 & 0.312 & 0.07 & -0.05 to 0.1 & 0.254 & 13 & 0.04 & -0.01 to 0.09 & 0.129 \\
\hline Amygdala $P<5 \mathrm{E}-08$ & CPD & 2 & 0.02 & -0.11 to 0.16 & 741 & - & - & - & - & - & - & 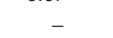 & - & - & - & - & - & - \\
\hline Amygdala $P<1 \mathrm{E}-05$ & CPD & 40 & -0.01 & -0.05 to 0.03 & 582 & 0.01 & -0.04 to 0.06 & 0.770 & 0.01 & -0.05 to 0.08 & 0.723 & 0.02 & -0.04 to 0.09 & 0.480 & 40 & -0.01 & -0.04 to 0.03 & 0.732 \\
\hline Caudate & CPD & 53 & $-6.1 \mathrm{E}-04$ & -0.04 to 0.04 & 0.974 & -0.02 & -0.06 to 0.02 & 0.250 & -0.04 & -0.13 to 0.04 & 0.322 & 1.9E-03 & -0.10 to 0.10 & 969 & 50 & -0.02 & -0.04 to 0.01 & 0.171 \\
\hline Hippocampus & CPD & 19 & 0.02 & -0.03 to 0.07 & & 0.03 & -0.02 to 0.09 & 246 & 0.04 & -0.03 to 0.1 & 0.302 & -0.05 & -0.24 to 0.13 & 575 & 19 & 0.03 & -0.01 to 0.06 & 0.177 \\
\hline Pallidum & CPD & 25 & 0.01 & -0.02 to 0.05 & 188 & -0.01 & -0.06 to 0.03 & 0.553 & -0.03 & -0.08 to 0.03 & 0.403 & 0.05 & -0.02 to 0 & 155 & 25 & 0.01 & -0.02 to 0.04 & 0.485 \\
\hline Putamen & CPD & 35 & $-6.9 \mathrm{E}-03$ & -0.04 to 0.02 & 664 & $2.8 \mathrm{E}-04$ & -0.04 to 0.04 & 889 & 0.01 & -0.04 to 0.06 & 0.636 & & -0.05 to $c$ & 14 & 34 & 1.4E-03. & -0.03 to 0.03 & 0.917 \\
\hline Thalamus & CPD & 12 & 0.03 & -0.07 to 0 & 41 & 0.04 & -0.05 to 0.12 & 0. & 0.02 & -0.13 to 0.1 & 0.802 & 0.08 & -0.19 to 0 & 569 & 11 & 0.06 & $0.00-0.12$ & 0.037 \\
\hline Nucleus & Alcohol & 13 & -0.01 & -0.12 to & & -0.06 & -0.22 to 0 . & 0.446 & -0.09 & -0.34 to 0 . & 0.514 & 0.26 & -0.06 to 0.57 & 0.134 & 13 & -0.01 & -0.12 to 0.10 & 0.884 \\
\hline Amygdala $P$ & Alcohol & 2 & 0.09 & -0.18 to 0.36 & 522 & - & - & - & - & - & - & - & - & - & - & - & - & - \\
\hline Amygdala $P$ & Alcohol & 39 & 0.08 & $1.2 \mathrm{E}-03$ to 0.0 & 0.046 & 0.10 & -0.01 to 0.22 & 0.081 & 0.09 & -0.04 to 0.21 & 0.193 & 0.01 & -0.15 to 0.16 & 0.916 & 39 & 0.08 & $0.00-0.15$ & 0.059 \\
\hline Caudate & Alcoho & 53 & 0.02 & -0.04 to $0 . c$ & & $3.2 \mathrm{E}-03$ & -0.08 to 0.09 & & $-6.5 E-03$ & -0.19 to 0.07 & 0.921 & -0.07 & -0.22 to 0.09 & .455 & 53 & 0.02 & -0.04 to 0.07 & 0.513 \\
\hline Hippocampus & Alcoho & 19 & 0.07 & & & & & & & -0.24 to 0.47 & & $0 . c$ & & & 19 & & -0.03 to 0.17 & 0.167 \\
\hline Pallidum & Alcohol d & 25 & 0.01 & -0.06 to 0.08 & 96 & -0.05 & -0.15 to 0.05 & & -0.04 & -0.16 to 0.09 & 0.560 & -0.05 & -0.21 to 0.10 & 508 & 25 & 0.01 & -0.06 to 0.08 & 0.796 \\
\hline Putamen & Alcohol d & 36 & -0.03 & -0.11 to 0.06 & & & -0.09 to 0.13 & & 0.04 & -0.07 to 0.16 & 0.4 & 0.02 & -0.20 to 0.23 & 0.870 & 35 & $-4.2 E-03$ & -0.07 to 0.06 & 0.900 \\
\hline Thalamus & Alcohol dependence & 13 & 0.03 & -0.13 to 0 & & 0.05 & -0.11 to 0.2 & & 0.08 & -0.14 to 0.3 & & 0.40 & -0.16 to 0 & 0.180 & 12 & 0.04 & -0.07 to 0.16 & 0.458 \\
\hline & DPW & 13 & $0 . c$ & -0.04 to 0 . & & $-3.6 \mathrm{E}-03$ & -0.04 to 0.03 & 0.856 & -0.01 & -0.07 to 0.04 & 0.616 & -0.15 & -0.28 to 0.02 & 0.039 & 11 & 0.01 & -0.02 to 0.04 & 0.456 \\
\hline & DPW & 2 & & -0.01 to 0.12 & & - & - & - & - & - & - & - & - & - & - & - & - & - \\
\hline Amygdala $P<1 \mathrm{E}-05$ & DPW & 40 & 0.01 & -0.01 to 0.03 & 0.289 & 0.01 & -0.01 to 0.04 & 0.388 & 0.01 & -0.03 to 0.05 & 0.520 & -0.04 & -0.08 to $0.7 E-03$ & 0.048 & 40 & 0.01 & -0.01 to 0.03 & 0.239 \\
\hline Caudate & DPW & 52 & $2.8 \mathrm{E}-03$ & -0.02 to 0.02 & & $-4.6 E-03$ & -0.02 to 0.02 & & $9.8 \mathrm{E}-03$ & -0.02 to 0.04 & 0.481 & 0.02 & -0.04 to 0.07 & 0.535 & 44 & -0.01 & -0.02 to 0.01 & 0.285 \\
\hline Hippocampus & DPV & 19 & 0.01 & -0.02 to 0.04 & 472 & 0.02 & -0.01 to 0.04 & 0.271 & 0.02 & -0.02 to 0.05 & 0.448 & -0.19 & -0.29 to 0.09 & 0.002 & 18 & $6.7 \mathrm{E}-04$ & -0.02 to 0.02 & 0.945 \\
\hline Pallidum & DPV & 25 & -0.01 & -0.04 to 0.01 & & -0.02 & -0.04 to $4.3 \mathrm{E}-03$ & 0.116 & -0.02 & -0.05 to $5.0 \mathrm{E}-0$ & 0.128 & -0.03 & -0.08 E 0.03 & 0.326 & 23 & -0.02 & -0.03 to 0.00 & 0.049 \\
\hline Putamen & DPW & 34 & $-5.5 E-03$ & -0.02 to 0.01 & 0.576 & $-4.9 \mathrm{E}-03$ & -0.02 to 0.01 & 0.628 & $-2.3 \mathrm{E}-03$ & -0.02 to 0.02 & 0.839 & -0.01 & -0.07 to 0.05 & 0.742 & 33 & -0.01 & -0.02 to 0.01 & 0.200 \\
\hline Thalamus & DPW & 13 & $1.0 \mathrm{E}-03$ & -0.04 to 0.04 & 0.957 & -0.01 & -0.04 to 0.03 & 0.701 & -0.01 & -0.07 to 0.05 & 0.740 & 0.18 & $0.09-0.28$ & 0.002 & 13 & $-1.4 \mathrm{E}-03$ & -0.03 to 0.02 & 0.915 \\
\hline
\end{tabular}

SNP, Single nucleotide polymorphism; IWW, inverse-variance weighted regression analysis; GSMR, generalised summary level Mendelian randomisation; CPD, cigarettes smoked per day; DPW, alcoholic drinks per week.
a. MR-Egger reported simulation extrapolation (SIMEX)-corrected values if the IGX $X^{2}$ statistic (regression dilution) was $<0.9$, or, if the IGX2 statistic was $<0.6$ nothing was reported. If the number of SNPS included in the $P<5 E-08$ instrument was $<10$, a lowered SNP inclusion a. MR-Egger reported simulation extrapolat
threshold $(P<1 E-05)$ was also reported. 


\begin{tabular}{|c|c|c|c|c|c|c|c|c|c|c|c|c|c|c|c|c|c|c|}
\hline \multirow[b]{2}{*}{ Exposure } & \multirow[b]{2}{*}{ Outcome } & \multirow{2}{*}{$\begin{array}{c}\text { SNP } \\
n\end{array}$} & & \multicolumn{2}{|l|}{ IVW } & \multicolumn{3}{|c|}{ Weighted median } & \multicolumn{3}{|c|}{ Weighted mode } & \multicolumn{3}{|c|}{ MR-Egger ${ }^{\mathrm{a}}$} & \multirow{2}{*}{$\begin{array}{l}\text { SNP } \\
\text { e } n\end{array}$} & \multicolumn{3}{|c|}{ GSMR } \\
\hline & & & $\beta$-value & $95 \% \mathrm{Cl}$ & $P$-value & $\beta$-value & $95 \% \mathrm{Cl}$ & $P$-value & $\beta$-value & $95 \% \mathrm{Cl}$ & $P$-value & $\beta$-value & $95 \% \mathrm{Cl}$ & $P$-value & & $\beta$-value & $95 \% \mathrm{Cl}$ & $P$-value \\
\hline Smoking initiation & $\begin{array}{l}\text { Nucleus } \\
\text { accumbens }\end{array}$ & 346 & -0.04 & -0.10 to 0.01 & 0.114 & -0.03 & -0.10 to 0.04 & 0.381 & -0.02 & -0.13 to 0.09 & 0.743 & - & - & - & 339 & -0.05 & -0.09 to 0.00 & 0.050 \\
\hline Smoking initiation & Amygdala & 346 & -0.05 & -0.07 to $-9.3 \mathrm{E}-04$ & 40.046 & 4.0E-04 & $4-0.07$ to 0.07 & 0.991 & 0.01 & -0.11 to 0.09 & 0.856 & - & - & - & 340 & -0.05 & -0.09 to 0.00 & 0.058 \\
\hline Smoking initiation & Caudate & 346 & $3.33 \mathrm{E}-03$ & $3-0.10$ to 0.07 & 0.919 & -0.02 & -0.10 to 0.06 & 0.660 & $-6.0 \mathrm{E}-03$ & -0.14 to 0.13 & 0.929 & - & - & - & 335 & -0.01 & -0.06 to 0.04 & 0.671 \\
\hline Smoking initiation & Hippocampus & 346 & $-2.20 \mathrm{E}-03$ & $3-0.09$ to 0.05 & 0.939 & -0.02 & -0.09 to 0.06 & 0.680 & $6.51 E-04$ & $4-0.13$ to 0.13 & 0.992 & - & - & - & 340 & -0.02 & -0.07 to 0.03 & 0.449 \\
\hline Smoking initiation & Pallidum & 346 & -0.04 & -0.09 to 0.02 & 0.166 & -0.02 & -0.09 to 0.05 & 0.502 & -0.01 & -0.14 to 0.13 & 0.934 & - & - & - & 338 & -0.03 & -0.08 to 0.02 & 0.206 \\
\hline Smoking initiation & Putamen & 346 & 0.02 & -0.09 to 0.08 & 0.614 & -0.01 & -0.09 to 0.06 & 0.728 & -0.03 & -0.15 to 0.09 & 0.618 & - & - & - & 336 & 0.01 & -0.04 to 0.06 & 0.614 \\
\hline Smoking initiation & Thalamus & 345 & -0.03 & -0.09 to 0.03 & 0.304 & -0.02 & -0.09 to 0.06 & 0.640 & -0.01 & -0.13 to 0.12 & 0.900 & - & - & - & 340 & -0.01 & -0.06 to 0.04 & 0.666 \\
\hline Alcohol dependence & $\begin{array}{l}\text { Nucleus } \\
\text { accumbens }\end{array}$ & 10 & -0.06 & -0.21 to 0.05 & 0.301 & -0.06 & -0.21 to 0.08 & 0.388 & -0.06 & -0.26 to 0.13 & 0.545 & -0.32 & $2-0.77$ to 0.12 & 0.189 & 10 & -0.07 & -0.20 to 0.06 & 0.309 \\
\hline Alcohol dependence & e Amygdala & 10 & -0.15 & -0.31 to -0.04 & 0.007 & -0.17 & -0.31 to -0.04 & 0.013 & -0.17 & -0.34 to -0.01 & 0.074 & -0.28 & $8-0.60$ to 0.05 & 0.276 & 10 & -0.15 & -0.28 to -0.02 & 0.023 \\
\hline Alcohol dependence & e Caudate & 10 & 0.04 & -0.14 to 0.16 & 0.552 & $2.1 \mathrm{E}-03$ & $3-0.14$ to 0.14 & 0.977 & 0.01 & -0.17 to 0.18 & 0.934 & 0.49 & $9-0.02$ to 1.01 & 0.099 & 10 & 0.02 & -0.11 to 0.15 & 0.734 \\
\hline Alcohol dependence & Hippocampus & 10 & -0.11 & -0.26 to -0.01 & 0.037 & -0.11 & -0.26 to 0.04 & 0.156 & -0.08 & -0.28 to 0.11 & 0.417 & -0.50 & $0-0.87$ to -0.13 & 0.031 & 10 & -0.12 & -0.25 to 0.01 & 0.077 \\
\hline Alcohol dependence & Pallidum & 10 & -0.02 & -0.13 to 0.11 & 0.756 & 0.03 & -0.13 to 0.18 & 0.737 & 0.12 & -0.07 to 0.32 & 0.252 & -0.50 & $0-1.08$ to 0.08 & 0.130 & 10 & 0.01 & -0.12 to 0.14 & 0.883 \\
\hline Alcohol dependence & e Putamen & 10 & -0.03 & -0.16 to 0.08 & 0.624 & -0.01 & -0.16 to 0.13 & 0.874 & -0.02 & -0.21 to 0.16 & 0.806 & 0.13 & $3-0.37$ to 0.62 & 0.630 & 10 & -0.01 & -0.14 to 0.12 & 0.891 \\
\hline Alcohol dependence & Thalamus & 10 & -0.09 & -0.21 to 0.02 & 0.097 & -0.06 & -0.21 to 0.09 & 0.443 & -0.03 & -0.21 to 0.15 & 0.774 & -0.46 & $6-0.91$ to $-3.7 \mathrm{E}-03$ & $3 \quad 0.084$ & 10 & -0.01 & -0.14 to 0.12 & 0.891 \\
\hline DPW & $\begin{array}{l}\text { Nucleus } \\
\text { accumbens }\end{array}$ & 92 & -0.13 & -0.39 to 0.04 & 0.122 & -0.18 & -0.39 to 0.04 & 0.111 & -0.17 & -0.38 to 0.05 & 0.137 & -0.18 & $8-0.46$ to 0.10 & 0.200 & 86 & -0.13 & -0.26 to 0.01 & 0.070 \\
\hline DPW & Amygdala & 92 & -0.11 & -0.46 to 0.04 & 0.147 & -0.25 & -0.46 to -0.03 & 0.025 & -0.24 & -0.48 to $-2.1 \mathrm{E}-04$ & 0.053 & -0.17 & $7-0.42$ to 0.07 & 0.174 & 87 & -0.13 & -0.27 to 0.00 & 0.057 \\
\hline DPW & Caudate & 92 & 0.20 & -0.06 to 0.39 & 0.032 & 0.18 & -0.06 to 0.41 & 0.138 & 0.09 & -0.12 to 0.31 & 0.401 & 0.14 & $4-0.16$ to 0.45 & 0.361 & 85 & 0.14 & $0.00-0.28$ & 0.054 \\
\hline DWP & Hippocampus & 92 & $-4.67 \mathrm{E}-03$ & $3-0.34$ to 0.14 & 0.949 & -0.14 & -0.34 to 0.06 & 0.170 & -0.18 & -0.40 to 0.03 & 0.100 & -0.10 & $0-0.33$ to 0.14 & 0.411 & 87 & -0.03 & -0.17 to 0.10 & 0.635 \\
\hline DPW & Pallidum & 92 & -0.15 & -0.52 to 0.03 & 0.096 & -0.29 & -0.52 to -0.05 & 0.017 & -0.31 & -0.53 to -0.09 & 0.007 & -0.39 & $9-0.68$ to -0.10 & 0.010 & 87 & -0.17 & -0.31 to -0.04 & 0.013 \\
\hline DPW & Putamen & 92 & -0.01 & -0.29 to 0.19 & 0.896 & -0.06 & -0.29 to 0.17 & 0.605 & -0.13 & -0.36 to 0.10 & 0.258 & -0.12 & $2-0.45$ to 0.21 & 0.482 & 83 & -0.01 & -0.15 to 0.13 & 0.839 \\
\hline DPW & Thalamus & 92 & -0.13 & -0.48 to 0.03 & 0.104 & -0.26 & -0.48 to -0.04 & 0.022 & -0.27 & -0.50 to -0.04 & 0.025 & -0.21 & $1-0.46$ to 0.05 & 0.118 & 86 & -0.13 & -0.27 to 0.00 & 0.058 \\
\hline CPD $D^{\text {Smokers }}$ & $\begin{array}{l}\text { Nucleus } \\
\text { accumbens }\end{array}$ & 49 & 2.28 & -8.14 to 12.71 & 0.668 & -1.41 & -14.32 to 11.51 & 0.831 & 2.75 & -7.42 to 12.92 & 0.598 & 9.95 & $5-5.16$ to 25.07 & 0.203 & 45 & 6.77 & -0.07 to 19.43 & 0.294 \\
\hline $\mathrm{CPD}^{\text {Smokers }}$ & Amygdala & 49 & 8.41 & -14.10 to 30.91 & 0.464 & 12.86 & -14.93 to 40.65 & 0.365 & 7.27 & -17.02 to 31.56 & 0.560 & 5.02 & $2-28.22$ to 38.25 & 0.769 & 45 & 5.62 & -10.38 to 35.2 & 0.710 \\
\hline $\mathrm{CPD}^{\text {Smokers }}$ & Caudate & 49 & -18.59 & -60.66 to 23.48 & 0.386 & -23.17 & -71.06 to 24.72 & 0.343 & -30.68 & -74.45 to 13.09 & 0.176 & -59.16 & $6-119.27$ to 0.95 & 0.060 & 44 & -13.30 & -41.1 to 38.09 & 0.612 \\
\hline $\mathrm{CPD}^{\text {Smokers }}$ & Hippocampus & 49 & -94.73 & -133.57 to -55.89 & $1.8 \mathrm{E}-06$ & -121.36 & -170.28 to -72.43 & 3 1.2E-06 & -108.97 & -153.96 to -63.99 & $1.9 \mathrm{E}-05$ & -106.98 & $8-164.19$ to -49.77 & 0.001 & 45 & -46.57 & -75.96 to 7.78 & 0.093 \\
\hline $\mathrm{CPD}^{\text {Smokers }}$ & Pallidum & 49 & -13.43 & -44.75 to 17.89 & 0.401 & -21.67 & -51.32 to 7.98 & 0.152 & -23.51 & -50.45 to 3.44 & 0.094 & -1.89 & $9-47.95$ to 44.18 & 0.936 & 45 & 7.19 & -8.43 to 36.07 & 0.626 \\
\hline $\mathrm{CPD}^{\text {Smokers }}$ & Putamen & 49 & -68.86 & -125.68 to -12.05 & 0.018 & -56.36 & -119.91 to 7.18 & 0.082 & -41.29 & -90.27 to 7.69 & 0.105 & -7.59 & $9-88.06$ to 72.87 & 0.854 & 45 & -30.52 & -64.02 to 31.43 & 0.334 \\
\hline $\mathrm{CPD}^{\text {Smokers }}$ & Thalamus & 49 & -1.95 & -55.21 to 51.30 & 0.943 & 27.72 & -40.07 to 95.51 & 0.423 & 0.60 & -54.88 to 56.07 & 0.983 & -5.23 & $3-83.93$ to 73.47 & 0.897 & 44 & 22.31 & -13.84 to 89.18 & 0.513 \\
\hline $\mathrm{CPD}^{\text {Neversmokers }}$ & $\begin{array}{l}\text { Nucleus } \\
\text { accumbens }\end{array}$ & 49 & -9.50 & -17.86 to -1.14 & 0.026 & -11.83 & -21.82 to -1.83 & 0.020 & -12.70 & -21.59 to -3.82 & 0.007 & -16.20 & $0-28.26$ to -4.14 & 0.011 & 45 & -5.72 & -15.86 to 4.43 & 0.269 \\
\hline $\mathrm{CPD}^{\text {Neversmokers }}$ & Amygdala & 49 & -15.66 & -34.05 to 2.73 & 0.095 & -20.19 & -42.69 to 2.30 & 0.079 & -19.79 & -39.25 to -0.33 & 0.052 & -20.93 & $3-48.02$ to 6.15 & 0.136 & 44 & 4.83 & -18.75 to 28.41 & 0.688 \\
\hline CPD ${ }^{\text {Neversmokers }}$ & Caudate & 49 & -12.17 & -50.53 to 26.19 & 0.534 & -8.08 & -52.37 to 36.21 & 0.721 & -18.29 & -53.10 to 16.52 & 0.308 & -36.42 & $2-92.27$ to 19.42 & 0.207 & 43 & 0.29 & -41.27 to 41.86 & 0.989 \\
\hline CPD ${ }^{\text {Neversmokers }}$ & Hippocampus & 49 & -30.40 & -60.75 to -0.04 & 0.050 & -26.96 & -66.70 to 12.79 & 0.184 & -24.92 & -62.12 to 12.28 & 0.195 & -9.42 & $2-53.78$ to 34.94 & 0.679 & 45 & -21.44 & -65.2 to 22.32 & 0.337 \\
\hline $\mathrm{CPD}^{\text {Neversmokers }}$ & Pallidum & 49 & -16.57 & -44.82 to 11.69 & 0.250 & -35.33 & -61.56 to -9.10 & 0.008 & -23.44 & -44.09 to -2.80 & 0.031 & -16.97 & $7-58.70$ to 24.76 & 0.429 & 43 & 11.91 & -14.08 to 37.9 & 0.369 \\
\hline CPD ${ }^{\text {Neversmokers }}$ & Putamen & 49 & -71.41 & -119.23 to -23.58 & 0.003 & -69.84 & -116.59 to -23.08 & 30.003 & -66.83 & -108.38 to -25.28 & 0.003 & -76.98 & $8-147.58$ to -6.37 & 0.038 & 44 & -53.17 & -101.94 to -4.39 & 0.033 \\
\hline $\mathrm{CPD}^{\text {Neversmokers }}$ & Thalamus & 49 & -45.30 & -92.05 to 1.46 & 0.058 & -67.14 & -117.07 to -17.20 & 0.008 & -52.49 & -92.41 to -12.57 & 0.013 & -65.05 & $5-133.66$ to 3.57 & 0.069 & 44 & -17.06 & -70.36 to 36.24 & 0.530 \\
\hline
\end{tabular}


respectively). Similarly, there was weak evidence for an effect of cigarettes per day on amygdala and thalamus volume in never smokers, pointing to horizontal pleiotropy.

There was evidence for a decreasing effect of liability to alcohol dependence on hippocampal volume $\left(\beta_{\mathrm{IVW}}=-0.11, P=0.037\right)$, and strong evidence for a decreasing effect on amygdala volume $\left(\beta_{\text {IVw }}=\right.$ $-0.15, P=0.007)$, consistent across weighted median, weighted mode, MR-Egger and GSMR (Supplementary Figs 3 and 4). There was weak evidence for horizontal pleiotropy for alcohol dependence to hippocampal volume (MR-Egger intercept $0.023, P=0.080$ ) and no clear evidence for alcohol dependence to amygdala volume (MREgger intercept $0.007, P=0.468)$. There was no clear evidence for heterogeneity in the SNP effects $(P=0.950$ and $P=0.691$, respectively). MR-PRESSO and Steiger filtering did not identify SNP outliers.

There was weak evidence that alcohol dependence decreases thalamus volume $\left(\beta_{\mathrm{IVW}}=-0.09, P=0.097\right)$, corroborated by MREgger, but not the other methods. There was no clear evidence for horizontal pleiotropy (MR-Egger intercept $0.021, P=0.150$ ) or heterogeneity $(P=0.493)$. No SNPs were excluded with MR-PRESSO or Steiger filtering.

There was evidence that more alcoholic drinks per week increases caudate volume $\left(\beta_{\mathrm{IVW}}=0.20, P=0.032\right)$. The effect was consistent with weighted median, but attenuated with weighted mode, MR-Egger and GSMR. There was no clear evidence for horizontal pleiotropy (MR-Egger intercept 0.001, $P=0.629$ ), but strong evidence for heterogeneity $(P=6.4 \mathrm{E}-10)$. MR-PRESSO identified five SNP outliers, which did not distort the causal estimate. Steiger filtering identified 26 SNPs that explained more variance in the outcome than the exposure and, after removing these (leaving 66 SNPs), no evidence for causality remained. Taken together, evidence that alcoholic drinks per week causally increases caudate volume is weak.

There was weak evidence that more alcoholic drinks per week decreases pallidum volume $\left(\beta_{\mathrm{IVW}}=-0.15, P=0.096\right)$, consistent and even stronger in size and statistical evidence across weighted median, weighted mode, MR-Egger and GSMR. There was evidence for horizontal pleiotropy (MR-Egger intercept $0.003, P=0.049$ ) and strong evidence for heterogeneity $(P=2.2 \mathrm{E}-08)$. MR-PRESSO identified two SNP outliers, but there was no distortion in the causal estimate after removal. With Steiger filtering, 35 SNPs were excluded (leaving 58 SNPs), but weak evidence for a causal effect remained.

Finally, from alcoholic drinks per week toamygdala and thalamus volumes, there were sizable negative effects. While the main IVW method provided no clear statistical evidence for causal relationships, the effects appeared much stronger in effect size and statistical evidence with the other sensitivity methods (Table 3 ). There was no horizontal pleiotropy (MR-Egger intercept $0.001, P=0.537$ and MR-Egger intercept $0.001, P=0.459$, respectively) but there was evidence for heterogeneity ( $P=0.005$ and $P=0.001$, respectively). There were no SNP outliers with MR-PRESSO and, although Steiger filtering excluded 27 and 25 SNPs, respectively, weak evidence for causality remained.

\section{Discussion}

This is the first Mendelian randomisation study to assess bi-directional, causal relationships between volume of subcortical brain regions and substance use behaviours. Our most robust findings indicated that liability to alcohol dependence causally decreases amygdala and hippocampal volume, and that smoking more cigarettes per day causally decreases hippocampal volume.

The evidence that alcohol dependence decreases amygdala and hippocampal volume is in line with findings that subcortical brain regions in individuals with alcohol dependence are smaller and have a lower thickness and surface area than in healthy controls (particularly the amygdala and hippocampus). ${ }^{9-11,14,36,37}$ Given Mendelian randomisation's powerful premise and the consistency of our findings across sensitivity analyses, we can make stronger conclusions that this is a result of causal effects. It had previously been hypothesised that alcohol can cause cell death/reduced cell density, resulting in volume loss. ${ }^{36}$ For instance, chronic alcohol consumption induces tumour necrosis factor alpha, a cytokine involved in potentiating neuroinflammation, which can cause neuronal death. ${ }^{38}$ When number of alcoholic drinks per week was the exposure, we found only weak evidence that more drinks decreases amygdala, pallidum and thalamus volumes, and no clear evidence for hippocampus volume. This discrepancy is likely, in part, because alcohol dependence is the more severe phenotype, reflecting prolonged and heavy exposure of the brain to alcohol. It may also be that downstream effects of alcohol dependence affect the brain, such as poor nutrition, social isolation or psychiatric comorbidity, rather than alcohol itself.

We found strong evidence that smoking more cigarettes (in smokers) decreases hippocampal volume and weak evidence that initiating smoking decreases amygdala volume, implying that smoking can induce structural subcortical brain changes. Although the literature on biological mechanisms responsible for such effects is scarce, animal models have shown that exposure to nicotine can induce apoptosis in hippocampal cells. ${ }^{39}$ In contrast to our findings, and to those of observational studies, ${ }^{4-7}$ a large ENIGMA study found smoking to be associated with greater thickness and surface area of subcortical regions. ${ }^{14}$ This discrepancy might be because the ENIGMA study was observational and influenced by confounders.

There is ongoing discussion whether differences in brain structure between substance users and controls reflect pre-existing differences, or are the result of alterations caused by substance use. Our results mostly point to the latter, with robust evidence for negative effects of liability to alcohol dependence and smoking on some subcortical volumes, without (similarly robust) evidence for causal effects from subcortical volumes to substance use. This is important knowledge, with potentially far-reaching consequences. Volume loss might lead to cognitive deficits and a higher chance of mental illness, since smaller amygdala and hippocampus volumes are implicated in psychiatric disorders. ${ }^{40}$ For instance, it is plausible that smoking-related structural brain changes in regions that connect fear response areas (e.g. amygdala) affect trait anxiety, subsequently leading to an anxiety disorder. ${ }^{41}$ More research is needed to test pathways from smoking and alcohol use to subcortical brain volume, and subsequently, psychiatric symptoms.

\section{Strengths and limitations}

This study has important strengths. We used large genetic data-sets, allowing us to test causal effects with sufficiently powered genetic instruments in both directions. We applied an extensive set of sensitivity methods to assess the robustness of our findings, allowing claims about causality with considerable certainty. It is unlikely that our findings were distorted by variations in explained variance (and thus statistical power) between instruments, given that the most robust evidence for causality was found with genetic instruments of comparably low explained variance. It is also unlikely that (genetic) overlap between smoking and alcohol use has affected our results, given that we uncovered distinctly different patterns of causal effects on (some) subcortical regions for smoking versus alcohol use, and that genetic correlations are low or moderate (alcoholic drinks per week with smoking initiation $\left(r_{g}=0.34\right)$ and with smoking cessation $\left(r_{g}=\right.$ -0.11) were Bonferroni-corrected significant; alcoholic drinks per week with cigarettes per day $\left(r_{g}=0.07\right)$ was not significant). ${ }^{19}$ 
There are, however, limitations to consider. The identified causal relationships may not exhaustively represent causal chains. Although many techniques were used to correct for horizontal pleiotropy, vertical pleiotropy (some other factor mediating the exposure) cannot be ruled out. In addition, because we used separate samples to obtain exposure and outcome estimates, we were unable to ascertain if individuals in the outcome sample were affected by said exposure (apart from cigarettes per day, for which we stratified on smoking status). Therefore, we should interpret all causal effects as an effect of the liability to that exposure. It is also important to note that causal effects found with Mendelian randomisation should be interpreted as lifetime exposures. ${ }^{42}$ Although we included adult participants and the GWAS were corrected for age (Supplementary Table 2), our results do not provide information on temporal patterns. In addition, it should be noted that our findings result from samples of European ancestry, which limits the interpretability in other populations. This is a common limitation in the field of genetics, as up to $2017, \sim 88 \%$ of GWAS studies were based on populations solely of European descent. ${ }^{43}$ Follow-up research with a higher geographic coverage would be highly recommended to investigate if our findings are mirrored in populations of non-European descent. Finally, although Mendelian randomisation can provide powerful causal inference, there may be bias from 'genetic nurturing' (the genotype of parents directly affecting offspring phenotypes even if the responsible variants are not transmitted), 'assortative mating' (spouses showing higher phenotypic and genotypic similarity than expected by chance) and geographic clustering. These phenomena reintroduce bias from confounding. When such data become available, future Mendelian randomisation studies should use genetic estimates from large-scale within-family GWAS, as these can correct for more fine-grained (geographical/family) clustering. ${ }^{4}$

In conclusion, we report robust evidence that liability to alcohol dependence causally affects the brain, decreasing subcortical volume (at least pertaining to the hippocampus and amygdala). For smoking there was strong evidence that it causally decreases hippocampal volume, and more tentatively, amygdala volume. These findings provide additional proof that smoking and alcohol use can be detrimental to the brain, and it may implicate structural changes as a pathway connecting substance use to the development of psychiatric disorders. We feel that, combined with accumulating evidence from other types of research, this justifies more recognition in public health efforts and clinical practice.

Emma Logtenberg, Department of Psychiatry, Amsterdam UMC, University of Amsterdam, The Netherlands; Martin F. Overbeek, Department of Psychiatry, Amsterdam UMC, University of Amsterdam, The Netherlands; Joëlle A. Pasman Behavioural Science Institute, Radboud University Nijmegen, The Netherlands; Abdel Abdellaoui, Department of Psychiatry, Amsterdam UMC, University of Amsterdam, The Netherlands; Maartje Luijten, Behavioural Science Institute, Radboud University Nijmegen, The Netherlands; Ruth J. van Holst, Department of Psychiatry, Amsterdam UMC, University of Amsterdam, The Netherlands; Jacqueline M. Vink, Behavioural Science Institute, Radboud University Nijmegen, The Netherlands; Damiaan Denys, Department of Psychiatry, Amsterdam UMC, University of Amsterdam, The Netherlands; Sarah E. Medland, Psychiatric Genetics Group, QIMR Berghofer Medical Research Institute, Australia; Karin J. H. Verweij, Department of Psychiatry, Amsterdam UMC, University of Amsterdam, The Netherlands; Jorien L. Treur (D), Department of Psychiatry, Amsterdam UMC, University of Amsterdam, The L. Treur $\mathbb{D}$, Deph

Correspondence: Jorien L. Treur. Email: j.I.treur@amsterdamumc.n

First received 11 Jan 2021, final revision 22 Apr 2021, accepted 13 May 2021

\section{Supplementary material}

To view supplementary material for this article, please visit https://doi.org/10.1192/bjp.2021.81

\section{Data availability}

The data that support the findings of this study include summary-level data of published GWAS, all of which are publicly available or can be requested from the corresponding author, J.L.T. Some of the analyses were based on data from UK Biobank, which cannot be provided by the authors but can be requested from UK Biobank directly.

\section{Acknowledgements}

This study used summary statistics provided by 23andMe. We would like to thank the research participants and employees of $23 a n d M e$ for making this work possible.

\section{Author contributions}

J.L.T. and K.J.H.V. conceived and designed the study. E.L. and M.F.O. conducted the data analyses, with help from J.L.T., A.A. and J.A.P.. J.L.T. wrote the manuscript, with significant contributions from E.L. and M.F.O.. M.L., R.J.V.-H., J.M.V., D.D. and S.E.M. contributed specific expert insights in neuro-imaging and clinical implications. All authors critically reviewed the report, proposed revisions and approved the final manuscript for publication.

\section{Funding}

J.L.T. is supported by a Veni grant from the Netherlands Organization for Scientific Research (NWO; grant number 016.Veni.195.016). M.L. is also supported by a Veni grant from the NWO (grant number 451-15-029). K.J.H.V., A.A. and J.L.T. are supported by the Foundation Volksbond Rotterdam. A.A. is supported by grant 849200011 from The Netherlands Organisation for Health Research and Development. S.E.M. was supported by National Health and Medical Research Council grants APP1103623, APP1158127 and APP1172917. We acknowledge SURFsara for the usage of the Lisa cluster computer (supported by NWO, grant number 15725).

\section{Declaration of interest}

None.

\section{References}

1 Koob GF, Volkow ND. Neurocircuitry of addiction. Neuropsychopharmacology 2010; 35: 217-38.

2 Prom-Wormley E, Maes HHM, Schmitt JE, Panizzon MS, Xian H, Eyler LT, et al. Genetic and environmental contributions to the relationships between brain structure and average lifetime cigarette use. Calcif Tissue Int 2015; 96: 157-70.

3 Effertz T, Mann K. The burden and cost of disorders of the brain in Europe with the inclusion of harmful alcohol use and nicotine addiction. Eur Neuropsychopharmacol 2013; 23: 742-8.

4 Das D, Cherbuin N, Anstey KJ, Sachdev PS, Easteal S. Lifetime cigarette smoking is associated with striatal volume measures. Addict Biol 2012; 17: 817-25.

5 Lin F, Han X, Wang Y, Ding W, Sun Y, Zhou Y, et al. Sex-specific effects of cigarette smoking on caudate and amygdala volume and resting-state functional connectivity. Brain Imaging Behav 2021; 15(1): 1-13.

6 Hanlon CA, Owens MM, Joseph JE, Zhu X, George MS, Brady KT, et al. Lower subcortical gray matter volume in both younger smokers and established smokers relative to non-smokers. Addict Biol 2016; 21: 185-95.

7 Hawkins KA, Emadi N, Pearlson GD, Taylor B, Khadka S, King D, et al. The effect of age and smoking on the hippocampus and memory in late middle age. Hippocampus 2018; 28: 846-9.

8 Li Y, Yuan K, Cai C, Feng D, Yin J, Bi Y, et al. Reduced frontal cortical thickness and increased caudate volume within fronto-striatal circuits in young adult smokers. Drug Alcohol Depend 2015; 151: 211-9.

9 Shim J-H, Kim Y-T, Kim S, Baek H-M. Volumetric reductions of subcortical structures and their localizations in alcohol-dependent patients. Front Neurol 2019; 10: 247

10 Dager AD, McKay DR, Kent JW, Curran JE, Knowles E, Sprooten E, et al. Shared genetic factors influence amygdala volumes and risk for alcoholism. Neuropsychopharmacology 2015; 40: 412-20.

11 Squeglia LM, Rinker DA, Bartsch H, Castro N, Chung Y, Dale AM, et al. Brain volume reductions in adolescent heavy drinkers. Dev Cogn Neurosci 2014; 9: 117-25.

12 Kühn S, Mascharek A, Banaschewski T, Bodke A, Bromberg U, Büchel C, et al. Predicting development of adolescent drinking behaviour from whole brain structure at 14 years of age. elife 2019; 8: e44056.

13 Fein G, Greenstein D, Cardenas VA, Cuzen NL, Fouche JP, Ferrett $H$, et al. Cortical and subcortical volumes in adolescents with alcohol dependence but without substance or psychiatric comorbidities. Psychiatry Res Neuroimaging 2013; 214: 1-8. 
14 Chye Y, Mackey S, Gutman BA, Ching CRK, Batalla A, Blaine S, et al. Subcortical surface morphometry in substance dependence: an ENIGMA addiction working group study. Addict Biol 2020: 25(6): e12830.

15 Modabbernia A, Reichenberg A, Ing A, Moser DA, Doucet GE, Artiges E, et al. Linked patterns of biological and environmental covariation with brain structure in adolescence: a population-based longitudinal study. Mol Psychiatry [Epub ahead of print] 22 May 2020. Available from: https://doi.org/10.1038/ S41380-020-0757-X

16 Smith G D, Ebrahim S. 'Mendelian randomization': can genetic epidemiology contribute to understanding environmental determinants of disease? Int J Epidemiol 2003; 32: 1-22.

17 Andrews S, Marcora E, Goate A. Causal associations between potentially modifiable risk factors and the Alzheimer's phenome: a Mendelian randomization study. Ann Neurol 2021; 89: 54-65.

18 Hibar DP, Stein JL, Renteria ME, Arias-Vasquez A, Desrivières S, Jahanshad N et al. Common genetic variants influence human subcortical brain structures. Nature 2015; 520: 224-9.

19 Liu M, Jiang Y, Wedow R, Li Y, Brazel DM, Chen F, et al. Association studies of up to 1.2 million individuals yield new insights into the genetic etiology of tobacco and alcohol use. Nat Genet 2019; 51(2): 237-44.

20 Walters RK, Polimanti R, Johnson EC, McClintick JN, Adams MJ, Adkins AE, et al. Transancestral GWAS of alcohol dependence reveals common genetic underpinnings with psychiatric disorders. Nat Neurosci 2018; 21: 1656-69.

21 Taylor AE, Burgess S, Ware JJ, Gage SH, Richards JB, Smith GD, et al. Investigating causality in the association between $25(\mathrm{OH}) \mathrm{D}$ and schizophrenia. Sci Rep 2016; 6: 26496.

22 Hemani G, Zheng J, Elsworth B, Wade KH, Haberland V, Baird D, et al. The MRBase platform supports systematic causal inference across the human phenome. elife 2018; 7: e34408.

23 Katikireddi SV, Green MJ, Taylor AE, Davey Smith G, Munafò MR. Assessing causal relationships using genetic proxies for exposures: an introduction to Mendelian randomization. Addiction 2018; 113: 764-4.

24 Davies NM, Holmes M V, Davey Smith G. Reading Mendelian randomisation studies: a guide, glossary, and checklist for clinicians. BMJ 2018; 362: k601.

25 Bowden J, Davey Smith G, Haycock PC, Burgess S. Consistent estimation in Mendelian randomization with some invalid instruments using a weighted median estimator. Genet Epidemiol 2016; 40: 304-14.

26 Hartwig FP, Smith GD, Bowden J. Robust inference in two-sample Mendelian randomisation via the zero modal pleiotropy assumption. Int J Epidemiol 2017 46: $1985-98$

27 Bowden J, Davey Smith G, Burgess S. Mendelian randomization with invalid instruments: effect estimation and bias detection through Egger regression. Int J Epidemiol 2015; 44: 512-25.

28 Bowden J, Del Greco MF, Minelli C, Davey Smith G, Sheehan NA, Thompson JR. Assessing the suitability of summary data for two-sample Mendelian randomization analyses using MR-Egger regression: the role of the 12 statistic. Int J Epidemiol 2016; 45(6): 1961-74.

29 Zhu Z, Zheng Z, Zhang F, Wu Y, Trzaskowski M, Maier R, et al. Causal associations between risk factors and common diseases inferred from GWAS summary data. Nat Commun 2018; 9: 224

30 Verbanck M, Chen CY, Neale B, Do R. Detection of widespread horizontal pleiotropy in causal relationships inferred from Mendelian randomization between complex traits and diseases. Nat Genet 2018; 50: 693-8.
31 Hemani G, Tilling K, Davey Smith G. Orienting the causal relationship between imprecisely measured traits using genetic instruments. PLOS Genet 2017; 13: e1007081.

32 Pasman JA, Verweij KJH, Gerring Z, Stringer S, Sanchez-Roige S, Treur JL, et al. GWAS of lifetime cannabis use reveals new risk loci, genetic overlap with psychiatric traits, and a causal influence of schizophrenia. Nat Neurosci 2018; 21: $1161-70$

33 Sterne JAC, Smith GD, Cox DR. Sifting the evidence-what's wrong with significance tests? BMJ 2001; 322: 226.

34 Wootton RE, Richmond RC, Stuijfzand BG, Lawn RB, Sallis HM, Taylor GMJ, et al. Evidence for causal effects of lifetime smoking on risk for depression and schizophrenia: a Mendelian randomisation study. Psychol Med 2020; 50(14): 2435-43.

35 Treur JL, Demontis D, Sallis H, Richardson T, Wiers R, Borglum A, et al. Investigating causal pathways between liability to ADHD and substance use, and liability to substance use and ADHD risk, using Mendelian randomization. Addict Biol 2021; 26(1): e12849.

36 Durazzo TC, Tosun D, Buckley S, Gazdzinski S, Mon A, Fryer SL, et al. Cortical thickness, surface area, and volume of the brain reward system in alcohol dependence: relationships to relapse and extended abstinence. Alcohol Clin Exp Res 2011; 35: 1187-200.

37 Grace S, Rossetti MG, Allen N, Batalla A, Bellani M, Brambilla P, et al. Sex differences in the neuroanatomy of alcohol dependence: hippocampus and amygdala subregions in a sample of 966 people from the ENIGMA Addiction Working Group. Transl Psychiatry 2021; 11(1): 156

38 Rao R, Topiwala A. Alcohol use disorders and the brain. Addiction 2020; 115(8): 1580-9.

39 Oliveira-da-Silva A, Vieira FB, Cristina-Rodrigues F, Filgueiras CC, Manhães AC, Abreu-Villaça Y. Increased apoptosis and reduced neuronal and glial densities in the hippocampus due to nicotine and ethanol exposure in adolescent mice. Int J Dev Neurosci 2009; 27: 539-48.

40 Kolesar TA, Bilevicius E, Wilson AD, Kornelsen J. Systematic review and metaanalyses of neural structural and functional differences in generalized anxiety disorder and healthy controls using magnetic resonance imaging. Neurolmage Clin 2019; 24: 102016.

41 Moylan S, Jacka FN, Pasco JA, Berk M. How cigarette smoking may increase the risk of anxiety symptoms and anxiety disorders: a critical review of biological pathways. Brain Behav 2013; 3: 302-26.

42 Labrecque JA, Swanson SA. Interpretation and potential biases of Mendelian randomization estimates with time-varying exposures. Am J Epidemiol 2019; 188: 231-8.

43 Abdellaoui A, Verweij KJH. Dissecting polygenic signals from genome-wide association studies on human behaviour. Nat Hum Behav [Epub ahead of print] 13 May 2021. Available from: https://doi.org/10.1038/s41562-021-01110-y.

44 Brumpton B, Sanderson E, Heilbron K, Hartwig FP, Harrison S, Vie GÅ, et al. Avoiding dynastic, assortative mating, and population stratification biases in Mendelian randomization through within-family analyses. Nat Commun 2020; 11(1): 3519 